\title{
Influence of Acute Normobaric Hypoxia on Hemostasis in Volunteers with and without Acute Mountain Sickness
}

\author{
Marc Schaber, ${ }^{1}$ Veronika Leichtfried, ${ }^{1}$ Dietmar Fries, ${ }^{2}$ Maria Wille, ${ }^{3}$ Hannes Gatterer, ${ }^{3}$ \\ Martin Faulhaber, ${ }^{3}$ Philipp Würtinger, ${ }^{4}$ and Wolfgang Schobersberger ${ }^{5}$ \\ ${ }^{1}$ Institute for Sports Medicine, Alpine Medicine and Health Tourism, University for Health Sciences, \\ Medical Informatics and Technology (UMIT), Eduard-Wallnöfer-Zentrum 1, 6060 Hall, Austria \\ ${ }^{2}$ Department for General and Surgical Critical Care Medicine, Medical University of Innsbruck, Anichstrasse 35, \\ 6020 Innsbruck, Austria \\ ${ }^{3}$ Department of Sports Science, Leopold Franzens University Innsbruck, Fürstenweg 185, 6020 Innsbruck, Austria \\ ${ }^{4}$ Central Institute for Medical and Chemical Laboratory Diagnosis, Medical University of Innsbruck, Anichstrasse 35, \\ 6020 Innsbruck, Austria \\ ${ }^{5}$ Institute for Sports Medicine, Alpine Medicine and Health Tourism, Tirol Kliniken Innsbruck and University for Health Sciences, \\ Medical Informatics and Technology (UMIT), Eduard-Wallnöfer-Zentrum 1, 6060 Hall, Austria
}

Correspondence should be addressed to Wolfgang Schobersberger; wolfgang.schobersberger@tirol-kliniken.at

Received 1 July 2015; Accepted 1 September 2015

Academic Editor: Saulius Butenas

Copyright (C) 2015 Marc Schaber et al. This is an open access article distributed under the Creative Commons Attribution License, which permits unrestricted use, distribution, and reproduction in any medium, provided the original work is properly cited.

\begin{abstract}
Introduction. The aim of the present study was to investigate whether a 12-hour exposure in a normobaric hypoxic chamber would induce changes in the hemostatic system and a procoagulant state in volunteers suffering from acute mountain sickness (AMS) and healthy controls. Materials and Methods. 37 healthy participants were passively exposed to $12.6 \% \mathrm{FiO}_{2}$ (simulated altitude hypoxia of 4,500 m). AMS development was investigated by the Lake Louise Score (LLS). Prothrombin time, activated partial thromboplastin time, fibrinogen, and platelet count were measured and specific methods (i.e., thromboelastometry and a thrombin generation test) were used. Results. AMS prevalence was 62.2\% (LLS cut off of 3). For the whole group, paired sample $t$-tests showed significant increase in the maximal concentration of generated thrombin. ROTEM measurements revealed a significant shortening of coagulation time and an increase of maximal clot firmness (InTEM test). A significant increase in maximum clot firmness could be shown (FibTEM test). Conclusions. All significant changes in coagulation parameters after exposure remained within normal reference ranges. No differences with regard to measured parameters of the hemostatic system between AMS-positive and -negative subjects were observed. Therefore, the hypothesis of the acute activation of coagulation by hypoxia can be rejected.
\end{abstract}

\section{Introduction}

Acute, high altitude exposure induces a large variety of adaptive mechanisms in the nonadapted human body. Currently, the main research focus has been on the physiology and pathophysiology of the cardiovascular, cerebral, and pulmonary systems, including maladaptation, in acute mountain sickness $[1,2]$. There have only been few studies with a special focus on hypoxia-induced changes of plasma coagulation, fibrinolysis, and platelet function.

Most information about acute hypoxia and hemostatic changes has been obtained by studies focusing on long-haul flights and travel thrombosis. However, these data are inconsistent [3-7] and the results cannot be easily transferred to high altitude physiology since hypoxia during aircraft travel is moderate (maximum corresponding to an altitude of 2,500 m) and sitting in a cramped position itself may be a central trigger for coagulation changes.

Even studies focusing on high altitude are nonuniform. Maher et al. [8] investigated several parameters of coagulation and platelet aggregation during simulated high altitude exposure $(4,400 \mathrm{~m})$ and found some parameters to be changed indicative for a coagulopathy. O'Brodovich et al. [9] reported hemostatic changes after acute exposure 
to hypobaric and normobaric hypoxia (inspired fraction of oxygen, $\mathrm{FiO}_{2}=0.11$ ), showing shortening of activated partial thromboplastin time (aPTT) and an increase in procoagulant plasma factor VIII:C-like activity. In contrast, Bärtsch et al. [10] could not show any changes in fibrin or thrombin formation during a 22-hour ascent from 3,200 m to 4,559 m. A prothrombotic state was reported by Mannucci et al. [11] in unacclimatized subjects who were transported by helicopter after a 48-hour stay from 1,200 $\mathrm{m}$ to 3,940 $\mathrm{m}$ and after another 24-hour stay were transported to $5,060 \mathrm{~m}$.

At high and extreme altitudes, subjects are exposed to a variety of factors which could influence the hemostatic system (e.g., cold, dehydration, polyglobulia, immobility during periods of bad weather, and exhaustive physical exercise). Since decades, thrombotic and thromboembolic events have been described in climbers [12-16]. However, all reports were either case reports or retrospective observations; therefore, the prevalence of high altitude associated thromboembolism remains unclear. In addition, several mountaineers suffering from thrombosis had individual risk factors (e.g., oral contraceptives, genetic mutations as factor V Leiden mutation, and prothrombin polymorphism). Therefore, the impact of hypoxia itself as an independent risk factor for thrombosis at high altitude is still a matter of debate [17-19].

Hypoxic chamber studies appear to be an effective and valid method to investigate acute mountain sickness (AMS), since AMS not only manifests in nonacclimatized trekkers and mountaineers who rapidly ascent to altitudes above $2,500 \mathrm{~m}$ [20] but is also a frequent health-related problem for subjects in hypoxic chamber studies [21, 22]. Nevertheless, the link between acute hypoxia, AMS formation, and hemostasis is still unknown. Bartsch et al. [23] showed that, after climbing an altitude of 4,559 $\mathrm{m}$, factor VIII procoagulant activity and von Willebrand factor antigen were increased in AMS-positive subjects, whereas Pichler Hefti et al. [24] were unable to detect any association between AMS scores and coagulation parameters.

To evaluate the effects on procoagulants by acute and chronic hypoxia $[9,25]$, standard laboratory tests like prothrombin time (PT) and aPTT seem to be inferior compared to methods like thromboelastography (TEG) [26] or thrombin generation [27]. In 2012, TEG was used for the first time at high altitude settings $(5,300 \mathrm{~m})$. After a 13 -day standardized ascent profile (from 2,800 $\mathrm{m}$ to 5,300 m), TEG results showed reduced coagulation activation in healthy volunteers [26]. However, studies under standardized acute hypoxic conditions using TEG and thrombin generation are still lacking. Therefore, in the present study, hemostasis was analyzed by applying TEG and thrombin generation during a simulated acute hypoxic setting, in which subjects reached altitudes similar to high altitude tours and where confounding variables like cold, dehydration, and prolonged immobility could be ruled out due to the study design.

We hypothesized that a 12 -hour sojourn in a hypoxic chamber corresponding to $4,500 \mathrm{~m}$ would provoke the activation of hemostasis in nonacclimatized healthy volunteers. In addition, we speculated that this coagulation activation is more pronounced in volunteers who develop AMS during hypoxia as compared to those who do not.

\section{Materials and Methods}

2.1. Participants. The present study was part of a large, simulated, high altitude project performed in Innsbruck, Austria. Parts of the project have already been published [21]. Participants were mainly recruited via advertisements on the homepage of the Austrian Alpine Association and information via the mailing list of the University of Innsbruck. Exclusion criteria were pregnancy, reported cardiovascular, respiratory, neurological, and psychiatric diseases, migraine, chronic headache, smoking, permanent residence at altitudes exceeding 1,000 m, an overnight stay at altitudes greater than $2,500 \mathrm{~m}$ in the previous month, or exposure above $2,500 \mathrm{~m}$ for 2 weeks prior to the 12-hour hypoxic exposure. Participants were instructed to abstain from all anti-inflammatory medications and nutritional supplements for 2 weeks prior to the exposure and from alcohol starting the day before the experiment. Caffeine was not allowed on the day of the exposure.

All participants gave their written informed consent prior to participation in the study. The study was carried out in conformity with the ethical standards laid down in the 2008 Declaration of Helsinki and was approved by the Ethics Committee of the Medical University of Innsbruck (program code: UN4522, session: 306/4.11).

2.2. Procedures. Participants were passively exposed to a $\mathrm{FiO}_{2}$ of $12.6 \%$ (corresponding to a simulated altitude hypoxia of $4,500 \mathrm{~m}$ at $590 \mathrm{~m}, \mathrm{PiO}_{2}=83.9 \mathrm{mmHg}$ ) for 12 hours. Room temperature and humidity were kept constant at $22-24^{\circ} \mathrm{C}$ and $23-27 \%$, respectively. Prior to entering the hypoxic chamber, participants were examined, including a medical routine check. During the stay in the chamber, food (e.g., brown bread, cheese, boiled ham, cucumber, banana, apple, cookies, and chocolate) and drinks (water and apple juice) were provided ad libitum. Most of the time, participants stayed seated, but some activities (e.g., standing, walking, and stretching) were also performed. Recumbent position or sleeping was not allowed.

\subsection{Measurements and Instruments}

2.3.1. Lake Louise Score (LLS) and AMS. To assess the prevalence and severity of AMS [28], the LLS was used. It is a selfassessment questionnaire including five symptom complexes (headache; gastrointestinal symptoms like anorexia, nausea, or vomiting; fatigue and/or weakness; dizziness and/or light headedness; and difficulty of sleeping); scores range from 0 to 3. The subjects self-rated their status: 0 for no discomfort and 1 for mild, 2 for moderate, and 3 for severe symptoms. Since participants did not stay overnight in the hypoxic chamber, the symptom complex "difficulty sleeping" was not taken into account. AMS was diagnosed when the symptom headache and at least one other symptom were present, with a total score of at least 3. Scores did not distinguish between mild and severe forms of AMS [29]. The LLS was assessed before entering the chamber and after 3, 6, 9, and 12 hours in the chamber or when participants left the chamber at an earlier time point. The maximum AMS score was used to distinguish 
between AMS-positive (AMS+) and AMS-negative (AMS-) volunteers.

Arterial oxygen saturation $\left(\mathrm{SpO}_{2}\right)$ and heart rate were measured using pulse oximetry (Onyx II 9550, NONIN, Plymouth, MI, USA) after $0.5,3,6,9$, and 12 hours in the chamber.

\subsubsection{Hematological Parameters, Thromboelastometry} (ROTEM), and Thrombin Generation. Venous blood samples were taken immediately before and at the end of the 12-hour hypoxic exposure or when participants left the chamber at an earlier time point. Blood processing was done immediately thereafter in the hemostasis laboratory.

Blood was drawn in ethylenediaminetetraacetic acidcontaining tubes (Sarstedt, Nümbrecht, Germany) for platelet counts and in sodium citrate-containing tubes (Sarstedt) for coagulation assays. Platelet counts were determined using the Sysmex XE 5000 hematology analyzer (Sysmex Corporation, Kobe, Japan).

Platelet-poor plasma for the determination of plasma coagulation tests was obtained by centrifugation at $2,100 \times \mathrm{g}$ for $15 \mathrm{~min}$ and another centrifugation at 10,000 $\times \mathrm{g}$ for $5 \mathrm{~min}$.

Parameters of aPTT, PT, and Clauss fibrinogen assay results [30] were determined on a BCS-XP instrument (Siemens Healthcare Diagnostics, Marburg, Germany) and an automated coagulation analyzer.

In ROTEM, the resistance of a rotated pin in a stationary cuvette, filled with citrated whole blood, is measured after coagulation activation with different reagents. For additional monitoring of routine coagulation tests in subjects, the following parameters were automatically detected by the ROTEM analysis software based on thromboelastograms: clotting time (CT), clot formation time (CFT), alpha angle, maximum clot firmness (MCF) after 15 min in ExTEM (activation of coagulation via tissue factor) and INTEM (activation of coagulation via the contact phase), and MCF after 15 min in FIBTEM (activation of coagulation via tissue factor and cytochalasin D for the inhibition of platelets). Although the ROTEM system was mainly established and used for the control and differential diagnosis of hemostatic disorders within the context of acute bleeding, recent literature has also suggested a possible role for the ROTEM system in testing for hypercoagulable states $[5,24]$. Additional information about ROTEM techniques, its parameters, and principles has been described in detail elsewhere [31-34].

Thrombin generation analysis was performed with platelet poor plasma using the Innovance ETP assay (Siemens) on a BCS XP automated coagulation analyzer (Siemens). After the activation of coagulation with synthetic phospholipids [35], human recombinant tissue factor (Innovin; BC Siemens), and calcium ions in the absence of thrombomodulin, a chromogenic substrate H-b-Ala-Gly-Arg-pNA is cleaved by the generated thrombin. The final concentration of substrate is $733 \mathrm{nmol} / \mathrm{L}$ and $19 \mathrm{mmol} / \mathrm{L} \mathrm{CaCl}$. The concentration of phospholipids and tissue factor is confidential to the manufacturer. To prevent fibrin polymerization, the reagent contains an undefined clot inhibitor. The conversion of the slow-reacting chromogenic substrate is detected over time at a wavelength of $405 \mathrm{~nm}$. After correction for the $\alpha$-macroglobulin-bound thrombin activity, the thrombin generation curve can be obtained. From this curve, the total amount of generated thrombin referred to as "endogenous thrombin potential" (area under the curve, ETP AUC), the peak thrombin generation $\left(C_{\max }\right)$, the lag phase until initiation $\left(t_{\mathrm{lag}}\right)$, and the time to peak thrombin activity $\left(t_{\max }\right)$ can be determined. The concentration of generated thrombin $\left(C_{\max }\right)(\mathrm{mE} / \mathrm{min})$ is the maximum of the first derivation of the ETP AUC. It corresponds with the maximal thrombin generation. These two values were calibrated against a normal human plasma pool (Innovance ETP Standard, BC Siemens) and then expressed as a percentage. The time needed until the first thrombin activity is registered is the lag time $\left(t_{\text {lag }}\right)(\mathrm{sec})$. The time to reach the maximum peak, which constitutes the maximum thrombin generation, is called $t_{\max }$ (sec) [36].

2.4. Statistical Analysis. All participants who endured the simulated exposure to high altitude for 12 hours without suffering severe symptoms were included in the statistical analysis. Others were counted as dropouts. Box plots were used to determine whether data distributions were symmetrical. Parametric tests were used for normally distributed data, and nonparametric tests were used for skew data. Results from categorical variables are reported as proportions, and continuous variables are reported as means \pm standard deviation (SD). Comparisons of parameters before and after simulated altitude exposure were made using the Student's $t$-test (normally distributed data) and Wilcoxon test (skew data) for matched pairs. Comparison of change scores (before hypoxia and after hypoxia) between participants suffering from AMS (LLS $\geq 3$; AMS+) and those not suffering from AMS (LLS < 3; AMS-) was made using the Student's $t$ test (normally distributed data) and Mann-Whitney $U$ test (skew data) for unmatched pairs. Spearman's rank correlation coefficients $\left(r_{\mathrm{S}}\right)$ of all blood parameters were evaluated.

Changes over time in heart rate and $\mathrm{SpO}_{2}$ values, as well as location of changes over time, were calculated using linear models for repeated measurements. Significant values were adapted using Bonferroni's correction. Two-tailed $P$ values less than 0.05 were determined to be significant for all statistical evaluations.

All statistical calculations were made using IBM SPSS Statistics, version 20.0 (Chicago, IL, USA).

\section{Results}

No serious or unexpected adverse events were observed during the chamber stay.

3.1. Anthropometric Data and Baseline Characteristics. In total, 37 participants were included in the statistical analysis (Table 1). Sixteen females and twenty-one males participated. The average age of all participants was $25.9 \pm 5.6$ years (range, 19 to 42 years). Body height varied from $160 \mathrm{~cm}$ to $197 \mathrm{~cm}$ (mean, $174 \pm 9 \mathrm{~cm}$ ) and body weight from $42.8 \mathrm{~kg}$ to $88.3 \mathrm{~kg}$ (mean, $67 \pm 11 \mathrm{~kg}$ ). Body mass index (BMI) was $22.0 \pm 2.3$ (range, 15.7 to 26.5). The amount of exercise performance per week was $8.1 \pm 4.7$ hours (range, 1-25 hours). Of the female participants, 33.3\% $(n=5)$ took oral contraceptives. 
TABle 1: Anthropometric data and baseline characteristics.

\begin{tabular}{|c|c|c|c|c|c|}
\hline & All $(n=37)$ & Male $(n=21)$ & Female $(n=16)$ & AMS- $(n=14)$ & $\mathrm{AMS}+(n=23)$ \\
\hline Age [y] & $25.9 \pm 5.6$ & $25.3 \pm 5.2$ & $26.7 \pm 6.1$ & $26.4 \pm 3.9$ & $25.6 \pm 6.5$ \\
\hline Body height $[\mathrm{cm}]$ & $174 \pm 9$ & $179 \pm 8$ & $168 \pm 5$ & $178 \pm 10$ & $172 \pm 7$ \\
\hline Body weight $[\mathrm{kg}]$ & $67 \pm 11$ & $73 \pm 9$ & $60 \pm 8$ & $69.7 \pm 13.2$ & $66.0 \pm 9.3$ \\
\hline BMI $\left[\mathrm{kg} / \mathrm{m}^{2}\right]$ & $22.0 \pm 2.3$ & $22.7 \pm 2.2$ & $21.2 \pm 2.2$ & $21.8 \pm 2.8$ & $22.2 \pm 1.9$ \\
\hline
\end{tabular}

Baseline characteristics of included participants after classification of those without AMS (AMS-) and those suffering from AMS symptoms (AMS+). Data are shown as mean values \pm standard deviation.

Both groups (AMS-/AMS+) were homogeneous, and no significant differences were shown for age $(P=0.690)$, body height $(P=0.072)$, or body weight $(P=0.375)$. AMS + group was comprised of eleven male and twelve female participants while the AMS- group included ten male and four female subjects.

3.2. AMS Prevalence. One participant who discontinued chamber exposure after 5 hours and $40 \mathrm{~min}$ was classified as a dropout, because he showed no symptoms of AMS and terminated for unknown reasons. Five subjects were excluded as dropouts as a result of preanalytical errors such as insufficient blood sample volumes or missing AMS data. Due to severe symptoms, nine participants (three male and six female subjects) left the chamber before the end of the 12 hours (mean LLS $=5.2 \pm 1.4$ ). The analysis of all included volunteers $(n=37)$ resulted in an AMS prevalence of $62.2 \%$ $(n=23$, on basis of the overall maximum AMS score of $\geq$ $3)$; 14 subjects $(37.8 \%)$ did not show any signs of AMS (in total minimum $\mathrm{LLS}=0$, maximum $\mathrm{LLS}=8$, and mean LLS $=$ $3.4 \pm 2.1 ; n=37)$. Of the 28 subjects who completed the full $12-$ hour session in the chamber, 19 subjects $(67.9 \%)$ were AMSand $9(32.1 \%)$ were AMS+ (minimum LLS $=0$, maximum LLS $=6$, and mean LLS $=1.79 \pm 1.89 ; n=28)$.

3.3. Peripheral Capillary Oxygen Saturation $\left(\mathrm{SpO}_{2}\right)$ and Heart Rate (HR). Whole-group data showed that both $\mathrm{HR}$ and $\mathrm{SpO}_{2}$ significantly changed over time (HR from $76 \pm 12 \mathrm{bpm}$ to $84 \pm 15 \mathrm{bpm}, P=0.008$, and $\mathrm{SpO}_{2}$ from $98.1 \pm 1.2 \%$ to $85.1 \pm$ $6.2 \%, P<0.000)$. For $\mathrm{SpO}_{2}$ values, a significant decrease occurred in the first one-half hour of hypoxic exposure $(P<$ 0.000 ) but showed no further decrease afterwards.

HR was significantly higher after 3 hours of hypoxic exposure compared to preexposure values $(P=0.026)$ and stayed constant thereafter. There was no difference in $\mathrm{HR}_{\max }$ values during hypoxic exposure between AMS+ and AMS - subjects $(P=0.251)$. Comparison of $\mathrm{SpO}_{2}\left(\mathrm{SpO}_{2 \max }-\right.$ $\mathrm{SpO}_{2 \text { min }}$ ) during the chamber exposure between AMS+ and AMS - subjects revealed no differences $(P=0.801)$.

\subsection{Standard Parameters of Hemostasis, Thrombin Generation, and Thromboelastometry}

3.4.1. aPTT and PT. All baseline data of the aPTT and PT analysis were within the reference range. There were no changes in aPTT for the whole group or for AMS+ and AMSsubjects during hypoxic exposure. PT was increased after the chamber sojourn in the AMS- group only. A comparison of the differences (data before and after the chamber session) of the two populations resulted in a significant $\triangle \mathrm{PT}(P=0.035$; AMS- $6.1 \pm 9.3 \%$; AMS+ $0.4 \pm 6.4 \%$ ).

3.4.2. Platelet Count and Fibrinogen. Pooled data did not show any changes in platelet counts or fibrinogen, and baseline data stayed within reference ranges (Table 2). In subjects with AMS, no differences in pre- or posthypoxic exposure values could be shown for either parameter. In contrast, a significant increase in platelet count was detected in participants without AMS after chamber exposure.

3.4.3. Thrombin Generation. All baseline data from the thrombin generation analysis were within the reference range (Table 3). With the exception of $C_{\max }$, where an increase in the whole group was observed, no significant changes were measured for any other thrombin generation parameters independent of subgroup before or after hypoxia.

3.4.4. ROTEM Measurements. ROTEM baseline data measurements changed within reference ranges (Table 4). When comparing baseline values of the AMS+ and AMS- groups, only the ROTEM parameters of MCF InTEM $(P=0.023)$ and MCF ExTEM $(P=0.029)$ showed significant differences between pre- and postexposure. After hypoxia in the InTEM analysis, significant shortening of CT $(\Delta \mathrm{CT}=-6.3 \pm 15.5 \mathrm{sec})$ and an increase in MCF $(\triangle \mathrm{MCF}=0.95 \pm 2.7 \mathrm{~mm})$ were found for the whole group. Furthermore, a significant increase in $\operatorname{MCF}(\triangle \mathrm{MCF}=0.87 \pm 2.4 \mathrm{~mm})$ could be shown in the FibTEM test. A comparison of the differences between the means (data before and after the chamber session) of the two populations showed significant differences for $\triangle \mathrm{MCF}$ InTEM $(P=0.023$; AMS- $2.21 \pm 2.5 \mathrm{~mm}$; AMS $+0.17 \pm 2.6 \mathrm{~mm})$ and $\triangle \mathrm{MCF}$ $\operatorname{ExTEM}(P=0.029$; AMS $-1.36 \pm 3.4 \mathrm{~mm}$; AMS $+-1.22 \pm$ $3.3 \mathrm{~mm})$. A tendency for a change in CFT ( $P=0.057$; AMS$-7.79 \pm 16.6 \mathrm{sec}$; AMS $+1.52 \pm 12.1 \mathrm{sec}$ ) in the InTEM analysis was observed.

The CT InTEM analysis showed a significant shortening in AMS+ subjects $(\triangle \mathrm{CT}=-7.1 \pm 12.2 \mathrm{sec})$, and MCF increased in AMS- subjects in both the InTEM $(\triangle \mathrm{MCF}=2.2 \pm 2.5 \mathrm{~mm})$ and FibTEM analyses $(\triangle \mathrm{MCF}=1.3 \pm 1.5 \mathrm{~mm})$.

3.5. Correlations. When assessing the relationship between LLS max and the laboratory parameters, a Spearman correlation coefficient of $0.329(P=0.046)$ between LLS max and $\triangle \mathrm{PT}$ was found. A negative Spearman correlation coefficient was measured for $\triangle$ CFT InTEM and LLS max $(-0.447 ; P=$ $0.006)$ and for $\triangle \mathrm{MCF}$ InTEM and LLS $\max (-0.413 ; P=$ 
TABLE 2: Levels of aPTT, PT, platelet count, fibrinogen, $\mathrm{SpO}_{2}$, and heart rate.

\begin{tabular}{|c|c|c|c|c|c|c|c|c|c|c|c|c|}
\hline & \multicolumn{4}{|c|}{ ALL } & \multicolumn{4}{|c|}{ AMS+ } & \multicolumn{4}{|c|}{ AMS- } \\
\hline & $n$ & Before & After & $P$ & $n$ & Before & After & $P$ & $n$ & Before & After & $P$ \\
\hline aPTT $(26-37 \mathrm{sec})$ & 37 & $31.5 \pm 3.7$ & $30.8 \pm 3.4$ & 0.091 & 23 & $30.9 \pm 3.5$ & $30.1 \pm 3.0$ & 0.100 & 14 & $32.5 \pm 3.9$ & $31.9 \pm 3.7$ & 0.489 \\
\hline PT (70-130\%) & 37 & $91.7 \pm 10.3$ & $94.3 \pm 11.2$ & 0.058 & 23 & $93.3 \pm 8.8$ & $93.7 \pm 8.5$ & 0.748 & 14 & $89.1 \pm 12.3$ & $95.2 \pm 15.1^{*}$ & 0.029 \\
\hline $\begin{array}{l}\text { Platelet count } \\
(150-380 \mathrm{G} / \mathrm{L})\end{array}$ & 37 & $226.0 \pm 43.6$ & $232.3 \pm 61.4$ & .340 & 23 & $233.5 \pm 36.7$ & $232.3 \pm 64.1$ & 0.908 & 14 & $213.8 \pm 52.2$ & $232.4 \pm 58.9^{*}$ & 0.000 \\
\hline $\begin{array}{l}\text { Fibrinogen } \\
(210-400 \mathrm{mg} / \mathrm{dL})\end{array}$ & 37 & $233.1 \pm 36.9$ & $238.6 \pm 41.0$ & 160 & 23 & $238.5 \pm 38.8$ & $246.2 \pm 45.3$ & 0.104 & 14 & $224.1 \pm 33.0$ & $226.2 \pm 30.3$ & 0.787 \\
\hline $\mathrm{SpO}_{2}(92-98 \%)$ & 37 & $98.1 \pm 1.2$ & $85.1 \pm 6.2$ & $0.000^{*}$ & 23 & $98.4 \pm 1.0$ & $85.3 \pm 6.9$ & 0.000 & 14 & $97.5 \pm 1.5$ & $84.1 \pm 6.2$ & 0.000 \\
\hline $\begin{array}{l}\text { Heart rate } \\
(72-77 \mathrm{bpm})\end{array}$ & 37 & $76 \pm 12$ & $84 \pm 15$ & $0.008^{*}$ & 23 & $79.8 \pm 14.3$ & $86.3 \pm 16.4$ & 0.011 & 14 & $72.6 \pm 7.0$ & $81.1 \pm 12.0$ & 0.042 \\
\hline
\end{tabular}

aPTT, PT, platelet count, and plasma fibrinogen concentration for all participants (ALL) and for volunteers with (AMS+) and without (AMS-) AMS before and after hypoxia. Data are shown as mean values \pm standard deviation.

* for $P<0.05$ as compared to pre exposure. Reference values are given in parentheses.

TABLE 3: Thrombin generation.

\begin{tabular}{lcccccccccccc}
\hline & \multicolumn{4}{c}{ ALL } & \multicolumn{3}{c}{ AMS+ } & \multicolumn{4}{c}{ AMS- } \\
& $n$ & Before & After & $P$ & $n$ & Before & After & $P$ & $n$ & Before & After & $P$ \\
\hline ETP AUC (76-107\%) & 37 & $89.2 \pm 10.3$ & $89.9 \pm 11.4$ & 0.430 & 23 & $90.1 \pm 11.0$ & $90.8 \pm 11.5$ & 0.474 & 14 & $87.9 \pm 9.4$ & $88.3 \pm 11.5$ & 0.734 \\
$C_{\max }(79-110 \%)$ & 37 & $94.0 \pm 11.9$ & $100.2 \pm 16.7$ & $0.019^{*}$ & 23 & $95.4 \pm 12.4$ & $101.7 \pm 17.1$ & 0.083 & 14 & $91.7 \pm 11.0$ & $97.8 \pm 16.5$ & 0.122 \\
$t_{\text {lag }}(19.6-25.6 \mathrm{sec})$ & 37 & $23.9 \pm 4.9$ & $23.3 \pm 3.8$ & 0.540 & 23 & $24.2 \pm 5.9$ & $23.8 \pm 4.4$ & 0.802 & 14 & $23.4 \pm 2.4$ & $22.5 \pm 2.4$ & 0.143 \\
$t_{\max }(50.8-72.0 \mathrm{sec})$ & 37 & $66.5 \pm 16.9$ & $60.5 \pm 7.7$ & n.a. & 23 & $67.6 \pm 18.2$ & $59.7 \pm 5.5$ & n.a. & 14 & $64.7 \pm 15.1$ & $61.8 \pm 9.8$ & 0.465 \\
\hline
\end{tabular}

n.a.: not applicable.

Endogenous thrombin potential (ETP AUC), maximum concentration of thrombin $\left(C_{\max }\right)$, time to peak $\left(t_{\max }\right)$, and lag-time $\left(t_{\mathrm{lag}}\right)$ for all participants (ALL) and for volunteers with (AMS+) and without (AMS-) AMS before and after hypoxia. Data are shown as mean values \pm standard deviation.

${ }^{*}$ for $P<0.05$ as compared to pre exposure. Reference values are given in parentheses.

0.011). Furthermore, a correlation between $\triangle \mathrm{MCF}$ ExTEM and LLS max was found $(0.399 ; P=0.014)$.

\section{Discussion}

The aim of the study at hand was to investigate possible hypoxia-induced changes in hemostasis in primary healthy volunteers during short-term (12 hours) exposure in a normobaric hypoxic chamber. Furthermore, it was hypothesized that subjects developing AMS would exhibit an activation of coagulation. However, during the 12-hour hypoxic exposure, only a few, small changes in the routine as well as in the specialized parameters of coagulation and fibrinolysis could be detected. Moreover, no significant differences in key parameters between volunteers who developed AMS and those who did not were measured.

The present chamber study simulated an altitude of $4,500 \mathrm{~m}$, which was sufficient to provoke AMS even within 12 hours. The overall prevalence of AMS was 62.2\%, which proved that our setting was adequate not only to investigate possible coagulation alterations for the whole group of participants but also to detect group differences (AMS+ versus AMS-). There are different approaches to investigate AMS and its consequences in controlled settings. Commonly used methods are chamber decompression to generate hypobaric hypoxia or adjustments for oxygen levels for normobaric hypoxia [37, 38]. MacInnies et al. [37] exposed 25 subjects to a partial pressure of inspired oxygen of $90 \mathrm{mmHg}(4,000 \mathrm{~m}$ equivalent) and found AMS prevalence of $84 \%$ and $56 \%$ during two separate, 12 -hour night sessions, which are similar to our results and those of other studies performed in highaltitude environments [39, 40].

Our unacclimatized participants showed reduced peripheral capillary $\mathrm{SpO}_{2}$ and increased $\mathrm{HR}$ in the chamber. These results are in accordance with others who reported an activation of the sympathetic nervous system during acute exposure to high altitude, which was evident not only during environmental exposure but also in hypobaric chambers [4143]. Faulhaber et al. [22] were able to demonstrate that $\mathrm{SpO}_{2}$ measurements after $30 \mathrm{~min}$ of hypoxic exposure have the potential to detect AMS-susceptible individuals. Karinen et al. [44] also showed that reduced $\mathrm{SpO}_{2}$ during resting and after exercise measured at altitudes of $3,500 \mathrm{~m}$ and $4,300 \mathrm{~m}$ seems to predict AMS at high altitudes. In a recent meta-analysis of 12 studies, a significant association between differences in $\mathrm{SpO}_{2}$ and the risk of developing AMS was reported [45]. However, $\mathrm{SpO}_{2}$ and $\mathrm{HR}$ did not differ during hypoxia between AMS+ and AMS- subjects; thus, $\mathrm{SpO}_{2}$ had no predictive value at least in our setting.

Currently, the majority of publications related to hemostatic alterations in hypoxia have not been based on high altitude but on travel medicine, that is, travel-related thromboembolism. Studies were performed either under simulated moderate hypoxic conditions or during situations of longdistance travel (flights or bus travel). The corresponding data are conflicting and results vary from unchanged coagulation 
TABLE 4: ROTEM measurements.

\begin{tabular}{lcccccccccccc}
\hline & \multicolumn{4}{c}{ ALL } & \multicolumn{3}{c}{ AL } & \multicolumn{3}{c}{ AMS+ } & \multicolumn{4}{c}{ AMS- } \\
& $n$ & Before & After & $P$ & $n$ & Before & After & $P$ & $n$ & Before & After & $P$ \\
\hline CT InTEM (134-218 sec) & 37 & $156.2 \pm 12.8$ & $149.9 \pm 16.6^{*}$ & 0.012 & 23 & $155.7 \pm 13.2$ & $148.6 \pm 14.4^{*}$ & 0.011 & 14 & $157.0 \pm 12.6$ & $152.1 \pm 20.0$ & 0.327 \\
CT ExTEM (42-78 sec) & 37 & $48.0 \pm 7.2$ & $49.8 \pm 9.8$ & 0.329 & 23 & $48.7 \pm 7.9$ & $50.2 \pm 11.6$ & 0.596 & 14 & $46.7 \pm 5.9$ & $49.1 \pm 6.2$ & 0.283 \\
CFT InTEM (52-116 sec) & 37 & $85.5 \pm 17.2$ & $83.5 \pm 20.5$ & 0.406 & 23 & $80.0 \pm 16.1$ & $81.5 \pm 18.6$ & 0.552 & 14 & $94.6 \pm 15.5$ & $86.8 \pm 23.7$ & 0.103 \\
CFT ExTEM (53-144 sec) & 37 & $107.6 \pm 22.3$ & $107.1 \pm 28.4$ & 0.884 & 23 & $99.4 \pm 18.2$ & $103.6 \pm 24.7$ & 0.325 & 14 & $121.2 \pm 22.3$ & $112.9 \pm 33.8$ & 0.237 \\
MCF InTEM (47-69 mm) & 37 & $54.8 \pm 4.2$ & $55.8 \pm 4.3^{*}$ & 0.039 & 23 & $55.7 \pm 4.4$ & $55.8 \pm 4.5$ & 0.749 & 14 & $53.5 \pm 3.7$ & $55.7 \pm 4.1^{*}$ & 0.005 \\
MCF ExTEM (48-70 mm) & 37 & $56.9 \pm 4.5$ & $56.6 \pm 5.1$ & 0.677 & 23 & $58.2 \pm 4.2$ & $57.0 \pm 5.0$ & 0.088 & 14 & $54.8 \pm 4.2$ & $56.1 \pm 5.6$ & 0.163 \\
MCF FibTEM (7-21 mm) & 37 & $11.6 \pm 2.4$ & $12.4 \pm 2.8^{*}$ & 0.035 & 23 & $12.2 \pm 2.4$ & $12.8 \pm 2.9$ & 0.307 & 14 & $10.6 \pm 2.3$ & $11.9 \pm 2.6^{*}$ & 0.008 \\
\hline
\end{tabular}

ROTEM analysis before and after chamber exposure for all volunteers (ALL) and according to absence (AMS-) or presence (AMS+) of AMS. ROTEM reference values are given in parentheses. Data are shown as mean values \pm standard deviation.

${ }^{*}$ for $P<0.05$ as compared to pre exposure.

$[6,7,46]$ to activation of coagulation and/or suppression of fibrinolysis $[3,4,47]$ in healthy subjects. A few studies even reported a reduced thrombin generation in hypoxia $[48,49]$. Although a few aspects of long-haul flights might be applicable to our study, the scientific approach is different. The degree of hypoxia is reduced during long-haul flights and in other chamber studies focusing on hemostasis compared to our study. In all travel-related studies, the participants were seated in a more-or-less cramped position that may worsen leg venous blood flow, thus triggering coagulation activation [50]. In our chamber study, the volunteers were able to sit comfortably and allowed to move freely during the chamber sojourn. Therefore, blood stasis in the lower legs can be excluded.

Data on hemostasis during real ambient hypoxia at high altitudes is scarce and more or less inconsistent. Only a few studies that focused on coagulation changes within a few hours of hypoxia are available. After a 22-hour ascent to $4,559 \mathrm{~m}$ Bärtsch et al. reported only a slight increase in PF1+2 with no evidence of significant thrombin or fibrin formation [10]. Similar results were obtained for TAT, PF1+2, and fibrinopeptide $\mathrm{A}$ in healthy mountaineers after a 2-3day walk to high altitudes [23]. In contrast, a prothrombotic state (increase in PF1+2 and PAI-1 activity and antigen) was reported in unacclimatized mountaineers after passive transport by helicopter from $1,200 \mathrm{~m}$ to $5,060 \mathrm{~m}$ within 2 days [11].

By pooling all data of the participants, independent of developing AMS, only a few significant changes in the measured hemostatic parameters were detected. In detail, in the thrombin generation analysis $C_{\max }$ was increased, the ROTEM CT InTEM was shortened, and MCF InTEM and MCF FibTEM were both increased. Recently, peak thrombin generation and ETP AUC were used as predictors of venous thromboembolism [51,52]. Additionally, in a cellbased model of coagulation, the influence of coagulation factors, which are involved in the formation of the tenase complex (factor (F)VIII, factor (F)IX, and factor (F)XI), on $C_{\max }$ was demonstrated [53]. Therefore, thrombin generation is thought to be an appropriate tool for the detection of hypercoagulation. Although the ROTEM system is currently established and used for the control and differential diagnosis of hemostatic disorders within the context of acute bleeding, recent literature has also suggested a possible role for the
ROTEM system in testing for hypercoagulable states [33, 34]. However, the absolute changes in the present study were small, and all parameters remained within the reference limits. The ROTEM results at hand are not consistent with those of Martin et al. [26], who reported reduced coagulation at high altitude identified by increased TEG reaction R-time (similar to CT) and kinetic K-time (similar to CFT). TEG and ROTEM are related tests where the only difference is in the use of the activator, but they do not have completely interchangeable results [54]. However, another reason for the incomparability of the data could be found in the differences of study design: in contrast to our study, the TEG analysis was performed after an ascent profile lasting for several days. Therefore, when interpreting pooled data, present results indicate that hypoxia does not trigger thrombin formation since the total amount of created free thrombin, as measured by ETP AUC which is indicative of maximal thrombin generation, remained unchanged. This finding was supported by the only minor changes in the thromboelastographic parameters.

In order to detect possible effects of hypoxia on AMS genesis, subgroup analyses were performed. In the AMS+ group, no changes in the standard coagulation tests aPTT, PT, platelet count, or fibrinogen were detected. In the AMSgroup, PT was shortened and platelet count was increased after hypoxia. ETP AUC remained unchanged in both groups during chamber exposure. Subgroup results of the ROTEM parameters showed shortening of CT InTEM in the AMS+ population and an increase of MCF in the InTEM and FibTEM analysis. Comparing the absolute changes between both groups, no stringent evidence for significant or relevant differences between AMS+ versus AMS- exists.

In case of a strong association between changes in hemostasis and the development of AMS, significant correlations of the maximum LLS during hypoxia with the measured coagulation parameters should have been obtained. However, only a few of the evaluated parameters showed a significant association with maximum LLS (PT, aPTT, CFT InTEM, MCF InTEM, and MCF ExTEM), and correlation coefficients were moderate to low $(<0.45)$ in all cases. This result might indicate the lack of a pathophysiological and clinical relationship between the development of AMS and hypercoagulation. 
Limitations of the present study include the relatively small number of participants $(N=37)$, short hypoxic exposure of 12 hours, and the impossibility to include sleep for calculating the original LLS. In addition, the volume of blood collection was limited for coagulation measurements, since it was also used for the determination of other laboratory parameters. This made the analysis of additional parameters for coagulation and fibrinolysis impossible. A further limitation may be the fact that our volunteers were passively exposed to hypoxia. They were allowed to move freely in the chamber, but no additional physical exercise was performed. Therefore, these data cannot be transferred to mountaineering, where both, hypoxia and physical exercise, are usually inseparable. Although it is well known that physical exercise has multiple effects on the hemostatic system, depending on type and intensity of exercise [55], there is no clear evidence that hypoxia per se exacerbates these acute exercise-dependent changes in hemostasis. For example, no differences between normoxic and hypoxic exercises (graded bicycle ergometry) were found for platelet-derived procoagulant microparticles, PDMP-mediated dynamic thrombin generation, and plasma coagulant factors TF, FV, and FVIII [56]. Even a suppression of normoxic exercise-induced increase of procoagulant factors during exercise in hypoxia was published [57].

\section{Conclusion}

In short, the hypothesis of a procoagulant effect of acute hypoxia in healthy individuals was not supported by the present study, since all data remained within normal reference ranges. Furthermore, a clinically relevant alteration of hemostasis in subjects suffering from AMS was not detected during exposure to hypoxia. Therefore, the authors conclude that there is no association in the development of AMS and hypercoagulability.

With respect to high altitude medicine, more studies need to be performed applying new hemostaseological methods that indicate in vivo thrombin formation during longer lasting high altitude exposure.

\section{Conflict of Interests}

The authors declare that there is no conflict of interests regarding the publication of this paper.

\section{Acknowledgments}

Marc Schaber was supported by a grant of the Austrian Society for Mountain Medicine (ÖGAHM). The authors thank the subjects who participated in their study and helped to expand the knowledge about high altitude medicine. The project was financially funded by the Oesterreichische Nationalbank.

\section{References}

[1] N. Netzer, K. Strohl, M. Faulhaber, H. Gatterer, and M. Burtscher, "Hypoxia-related altitude illnesses," Journal of Travel Medicine, vol. 20, no. 4, pp. 247-255, 2013.
[2] P. N. Ainslie, S. J. E. Lucas, and K. R. Burgess, "Breathing and sleep at high altitude," Respiratory Physiology and Neurobiology, vol. 188, no. 3, pp. 233-256, 2013.

[3] B. Bendz, M. Rostrup, K. Sevre, T. O. Andersen, and P. M. Sandset, "Association between acute hypobaric hypoxia and activation of coagulation in human beings," The Lancet, vol. 356, no. 9242, pp. 1657-1658, 2000.

[4] W. Schobersberger, D. Fries, M. Mittermayr et al., "Changes of biochemical markers and functional tests for clot formation during long-haul flights," Thrombosis Research, vol. 108, no. 1, pp. 19-24, 2002.

[5] W. Schobersberger, M. Mittermayr, D. Fries et al., "Changes in blood coagulation of arm and leg veins during a simulated longhaul flight," Thrombosis Research, vol. 119, no. 3, pp. 293-300, 2007.

[6] W. D. Toff, C. I. Jones, I. Ford et al., "Effect of hypobaric hypoxia, simulating conditions during long-haul air travel, on coagulation, fibrinolysis, platelet function, and endothelial activation," Journal of the American Medical Association, vol. 295, no. 19, pp. 2251-2261, 2006.

[7] A. Crosby, N. P. Talbot, P. Harrison, D. Keeling, and P. A. Robbins, "Relation between acute hypoxia and activation of coagulation in human beings," The Lancet, vol. 361, no. 9376, pp. 2207-2208, 2003.

[8] J. T. Maher, P. H. Levine, and A. Cymerman, "Human coagulation abnormalities during acute exposure to hypobaric hypoxia," Journal of Applied Physiology, vol. 41, no. 5, pp. 702707, 1976.

[9] H. M. O’Brodovich, M. Andrew, G. W. Gray, and G. Coates, "Hypoxia alters blood coagulation during acute decompression in humans," Journal of Applied Physiology: Respiratory, Environmental and Exercise Physiology, vol. 56, no. 3, pp. 666-670, 1984.

[10] P. Bärtsch, P. W. Straub, A. Haeberli et al., "Hypobaric hypoxia," The Lancet, vol. 357, no. 9260, pp. 955-956, 2001.

[11] P. M. Mannucci, A. Gringeri, F. Peyvandi, T. Di Paolantonio, and G. Mariani, "Short-term exposure to high altitude causes coagulation activation and inhibits fibrinolysis," Thrombosis and Haemostasis, vol. 87, no. 2, pp. 342-343, 2002.

[12] J. Dickinson, D. Heath, J. Gosney, and D. Williams, "Altituderelated deaths in seven trekkers in the Himalayas," Thorax, vol. 38, no. 9, pp. 646-656, 1983.

[13] T. Fujimaki, M. Matsutani, A. Asai, T. Kohno, and M. Koike, "Cerebral venous thrombosis due to high-altitude polycythemia. Case report," Journal of Neurosurgery, vol. 64, no. 1, pp. 148-150, 1986.

[14] S. Saito and S.-K. Tanaka, "A case of cerebral sinus thrombosis developed during a high-altitude expedition to Gasherbrum I," Wilderness and Environmental Medicine, vol. 14, no. 4, pp. 226230, 2003.

[15] S. C. Skaiaa and H. Stave, "Recurrent sagittal sinus thrombosis occurring at high altitude during expeditions to Cho Oyu," Wilderness and Environmental Medicine, vol. 17, no. 2, pp. 132136, 2006.

[16] A. Al Tahan, J. Buchur, F. El Khwsky et al., "Risk factors of stroke at high and low altitude areas in Saudi Arabia," Archives of Medical Research, vol. 29, no. 2, pp. 173-177, 1998.

[17] A. C. Anand, S. K. Jha, A. Saha, V. Sharma, and C. M. Adya, "Thrombosis as a complication of extended stay at high altitude," National Medical Journal of India, vol. 14, no. 4, pp. 197-201, 2001.

[18] C. P. Segler, "Prophylaxis of climbers for prevention of embolic accidents," Medical Hypotheses, vol. 57, no. 4, pp. 472-475, 2001. 
[19] N. Gupta and M. Z. Ashraf, "Exposure to high altitude: a risk factor for venous thromboembolism?" Seminars in Thrombosis and Hemostasis, vol. 38, no. 2, pp. 156-163, 2012.

[20] P. H. Hackett and R. C. Roach, "High-altitude illness," The New England Journal of Medicine, vol. 345, no. 2, pp. 107-114, 2001.

[21] H. Gatterer, M. Wille, M. Faulhaber et al., "Association between body water status and acute mountain sickness," PLOS ONE, vol. 8, no. 8, Article ID e73185, 2013.

[22] M. Faulhaber, M. Wille, H. Gatterer, D. Heinrich, and M. Burtscher, "Resting arterial oxygen saturation and breathing frequency as predictors for acute mountain sickness development: a prospective cohort study," Sleep and Breathing, vol. 18, no. 3, pp. 669-674, 2014.

[23] P. Bartsch, A. Haeberli, M. Franciolli, E. K. O. Kruithof, and P. W. Straub, "Coagulation and fibrinolysis in acute mountain sickness and beginning pulmonary edema," Journal of Applied Physiology, vol. 66, no. 5, pp. 2136-2144, 1989.

[24] J. Pichler Hefti, L. Risch, U. Hefti et al., "Changes of coagulation parameters during high altitude expedition," Swiss Medical Weekly, vol. 140, no. 7-8, pp. 111-117, 2010.

[25] M. Andrew, H. O’Brodovich, and J. Sutton, “Operation Everest II: coagulation system during prolonged decompression to 282 Torr," Journal of Applied Physiology, vol. 63, no. 3, pp. 1262-1267, 1987.

[26] D. S. Martin, J. S. Pate, A. Vercueil, P. W. Doyle, M. G. Mythen, and M. P. W. Grocott, "Reduced coagulation at high altitude identified by thromboelastography," Thrombosis and Haemostasis, vol. 107, no. 6, pp. 1066-1071, 2012.

[27] M. Wilkens, "Applications of the endogenous thrombin potential," Hamostaseologie, vol. 31, no. 2, pp. 88-93, 2011.

[28] R. C. Roach, P. Bartsch, O. Oelz, P. H. Hackett, and Lake Louise AMS Scoring Consensus Committee, "The Lake Louise acute mountain sickness scoring system," in Hypoxia and Molecular Medicine, J. R. Sutton, C. S. Houston, and G. Coates, Eds., pp. 272-274, Charles S. Houston, Burlington, Vt, USA, 1993.

[29] P. Bärtsch, D. M. Bailey, M. M. Berger, M. Knauth, and R. W. Baumgartner, "Acute mountain sickness: controversies and advances," High Altitude Medicine and Biology, vol. 5, no. 2, pp. 110-124, 2004.

[30] A. Clauss, "Gerinnungsphysiologische Schnellmethode zur Bestimmung des Fibrinogens," Acta Haematologica, vol. 17, no. 4, pp. 237-246, 1957.

[31] R. J. Luddington, "Thrombelastography/thromboelastometry," Clinical and Laboratory Haematology, vol. 27, no. 2, pp. 81-90, 2005.

[32] M. Chitlur and J. Lusher, "Standardization of thromboelastography: values and challenges," Seminars in Thrombosis and Hemostasis, vol. 36, no. 7, pp. 707-711, 2010.

[33] R. C. Franz, "ROTEM analysis: a significant advance in the field of rotational thrombelastography," South African Journal of Surgery, vol. 47, no. 1, pp. 2-6, 2009.

[34] M. S. Park, W. Z. Martini, M. A. Dubick et al., “Thromboelastography as a better indicator of hypercoagulable state after injury than prothrombin time or activated partial thromboplastin time," Journal of Trauma, vol. 67, no. 2, pp. 266-276, 2009.

[35] E. K. Spicer, R. Horton, L. Bloem et al., "Isolation of cDNA clones coding for human tissue factor: primary structure of the protein and cDNA," Proceedings of the National Academy of Sciences of the United States of America, vol. 84, no. 15, pp. 5148$5152,1987$.
[36] H. C. Hemker, R. Al Dieri, E. de Smedt, and S. Béguin, "Thrombin generation, a function test of the haemostaticthrombotic system," Thrombosis and Haemostasis, vol. 96, no. 5, pp. 553-561, 2006.

[37] M. J. MacInnies, S. Koch, K. E. Macleod et al., "Acute mountain sickness is not repeatable across two 12-hour normobaric hypoxia exposures," Wilderness \& Environmental Medicine, vol. 25, no. 2, pp. 143-151, 2014.

[38] R. C. Roach, J. A. Loeppky, and M. V. Icenogle, "Acute mountain sickness: increased severity during simulated altitude compared with normobaric hypoxia," Journal of Applied Physiology, vol. 81, no. 5, pp. 1908-1910, 1996.

[39] M. Santantonio, J.-M. Chapplain, P. Tattevin et al., "Prevalence of and risk factors for acute mountain sickness among a cohort of high-altitude travellers who received pre-travel counselling," Travel Medicine and Infectious Disease, vol. 12, no. 5, pp. 534540, 2014.

[40] S. Kriemler, F. Bürgi, C. Wick et al., "Prevalence of acute mountain sickness at $3500 \mathrm{~m}$ within and between families: a prospective cohort study," High Altitude Medicine and Biology, vol. 15, no. 1, pp. 28-38, 2014.

[41] L. Bernardi, C. Passino, G. Spadacini et al., "Cardiovascular autonomic modulation and activity of carotid baroreceptors at altitude," Clinical Science, vol. 95, no. 5, pp. 565-573, 1998.

[42] J. A. Vogel and C. W. Harris, "Cardiopulmonary responses of resting man during early exposure to high altitude," Journal of Applied Physiology, vol. 22, no. 6, pp. 1124-1128, 1967.

[43] R. Hainsworth, M. J. Drinkhill, and M. Rivera-Chira, "The autonomic nervous system at high altitude," Clinical Autonomic Research, vol. 17, no. 1, pp. 13-19, 2007.

[44] H. M. Karinen, J. E. Peltonen, M. Kähönen, and H. O. Tikkanen, "Prediction of acute mountain sickness by monitoring arterial oxygen saturation during ascent," High Altitude Medicine and Biology, vol. 11, no. 4, pp. 325-332, 2010.

[45] G. Guo, G. Zhu, W. Sun et al., "Association of arterial oxygen saturation and acute mountain sickness susceptibility: a metaanalysis," Cell Biochemistry and Biophysics, vol. 70, pp. 14271432, 2014.

[46] P. D. Hodkinson, B. J. Hunt, K. Parmar, and J. Ernsting, "Is mild normobaric hypoxia a risk factor for venous thromboembolism?" Journal of Thrombosis and Haemostasis, vol. 1, no. 10, pp. 2131-2133, 2003.

[47] W. Schobersberger, M. Mittermayr, P. Innerhofer et al., "Coagulation changes and edema formation during long-distance bus travel," Blood Coagulation and Fibrinolysis, vol. 15, no. 5, pp. 419425, 2004.

[48] H. Stricker, G. Colucci, L. Alberio, and G. Mombelli, "Variation in coagulation inhibitors during prolonged sitting: possible pathogenetic mechanisms for travel-associated thrombosis," Journal of Thrombosis and Haemostasis, vol. 4, no. 4, pp. 900902, 2006.

[49] H. Boccalon, B. Boneu, J. Emmerich, C. Thalamas, and J. B. Ruidavets, "Long-haul flights do not activate hemostasis in young healthy men," Journal of Thrombosis and Haemostasis, vol. 3, no. 7, pp. 1539-1541, 2005.

[50] K. Delis, A. L. Knaggs, T. N. Sonecha, V. Zervas, M. P. Jenkins, and J. H. N. Wolfe, "Lower limb venous haemodynamic impairment on dependency: quantification and implications for the 'economy class' position," Thrombosis and Haemostasis, vol. 91, no. 5, pp. 941-950, 2004. 
[51] P. L. Lutsey, A. R. Folsom, S. R. Heckbert, and M. Cushman, "Peak thrombin generation and subsequent venous thromboembolism: the Longitudinal Investigation of Thromboembolism Etiology (LITE) study," Journal of Thrombosis and Haemostasis, vol. 7, no. 10, pp. 1639-1648, 2009.

[52] M. Besser, C. Baglin, R. Luddington, A. van Hylckama Vlieg, and T. Baglin, "High rate of unprovoked recurrent venous thrombosis is associated with high thrombin-generating potential in a prospective cohort study," Journal of Thrombosis and Haemostasis, vol. 6, no. 10, pp. 1720-1725, 2008.

[53] G. A. Allen, A. S. Wolberg, J. A. Oliver, M. Hoffman, H. R. Roberts, and D. M. Monroe, "Impact of procoagulant concentration on rate, peak and total thrombin generation in a model system," Journal of Thrombosis and Haemostasis, vol. 2, no. 3, pp. 402-413, 2004.

[54] A. Sankarankutty, B. Nascimento, L. T. da Luz, and S. Rizoli, "TEG and ROTEM in trauma: similar test but different results?" World Journal of Emergency Surgery, vol. 7, supplement 1, article S3, 2012.

[55] J. J. Posthuma, P. E. van der Meijden, H. ten Cate, and H. M. Spronk, "Short- and Long-term exercise induced alterations in haemostasis: a review of the literature," Blood Reviews, vol. 29, no. 3, pp. 171-178, 2015.

[56] Y.-W. Chen, Y.-C. Chen, and J.-S. Wang, "Absolute hypoxic exercise training enhances in vitro thrombin generation by increasing procoagulant platelet-derived microparticles under high shear stress in sedentary men," Clinical Science, vol. 124, no. 10, pp. 639-649, 2013.

[57] T. G. DeLoughery, D. G. Robertson, C. A. Smith, and D. Sauer, "Moderate hypoxia suppresses exercise-induced procoagulant changes," British Journal of Haematology, vol. 125, no. 3, pp. 369$372,2004$. 

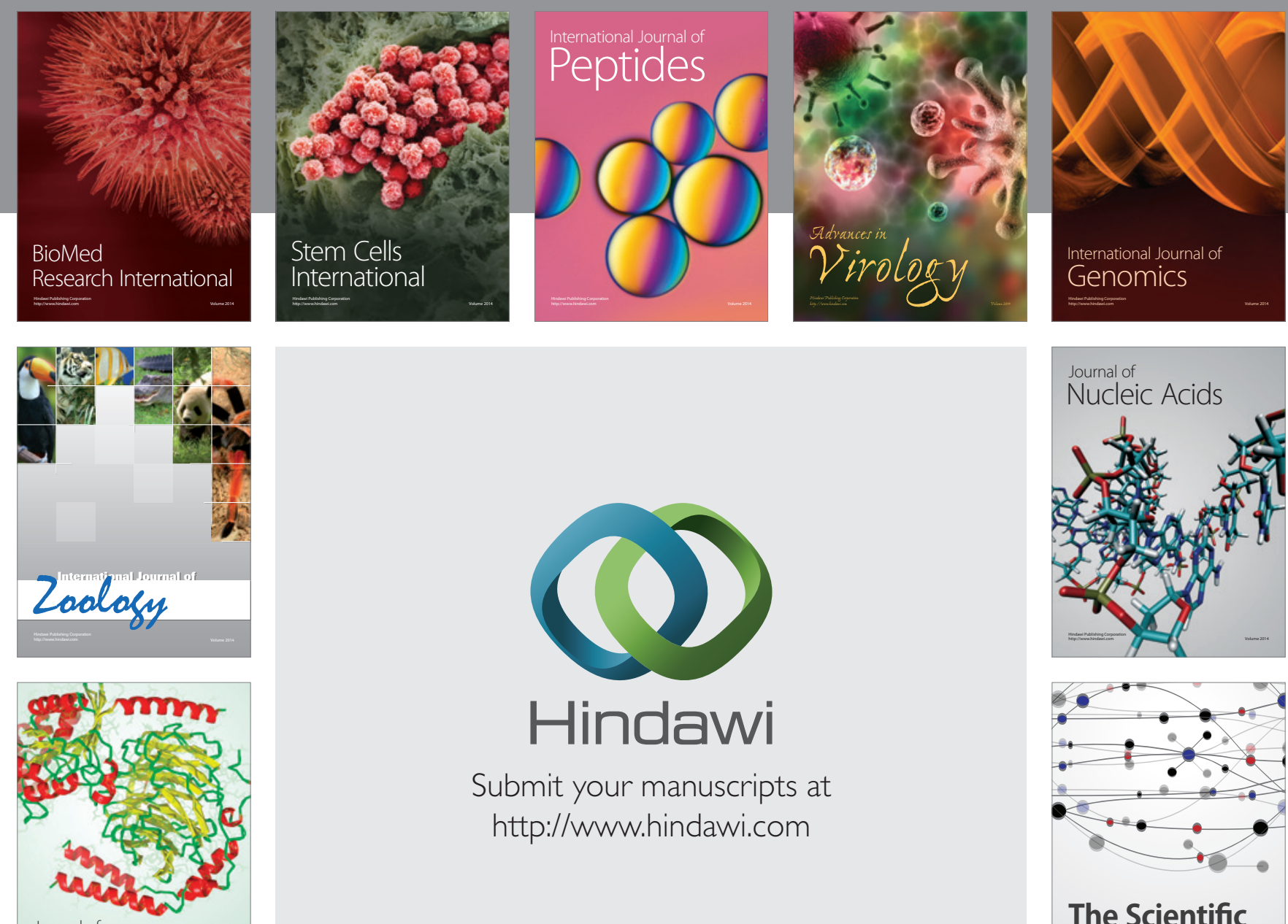

Submit your manuscripts at

http://www.hindawi.com

Journal of
Signal Transduction
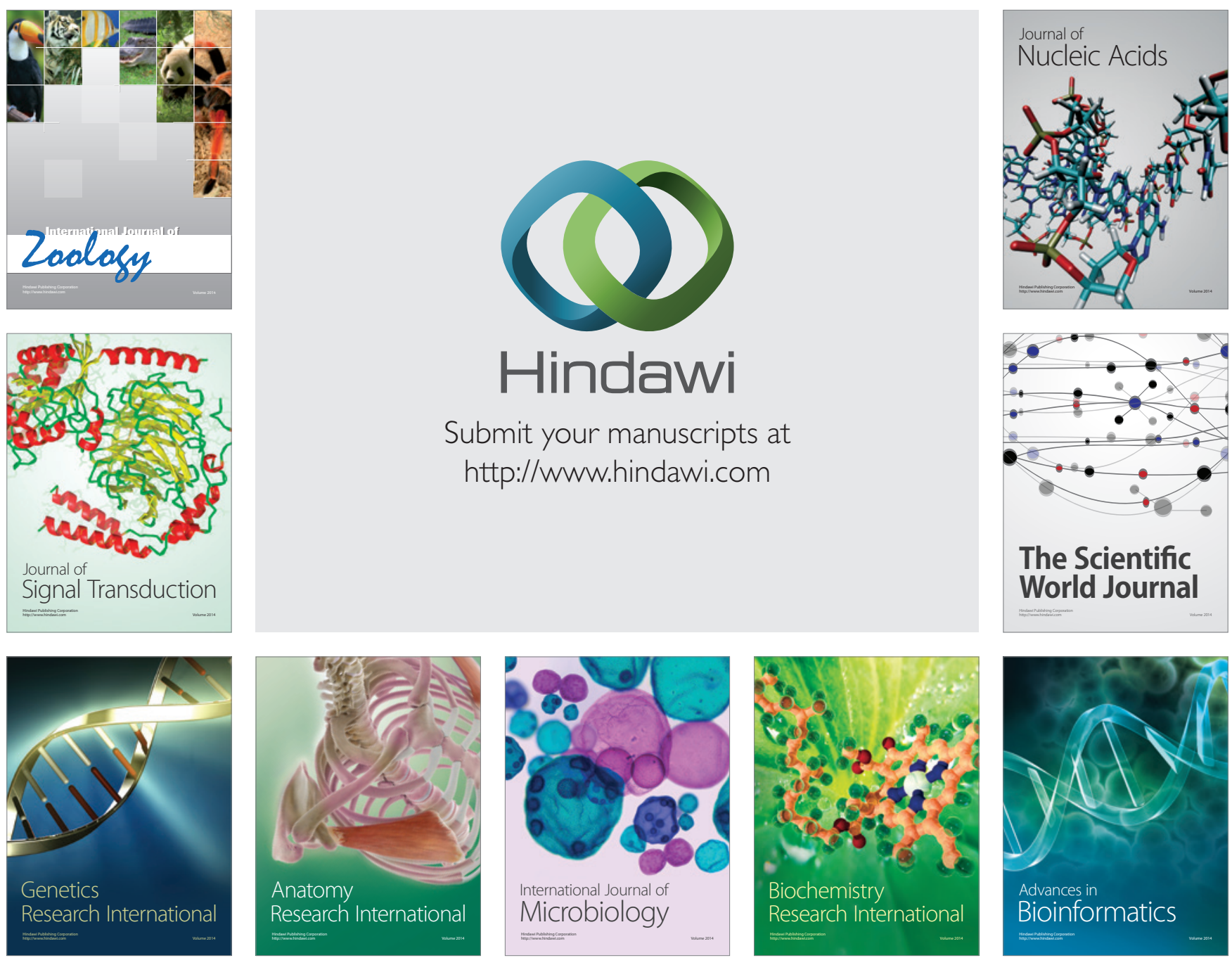

The Scientific World Journal
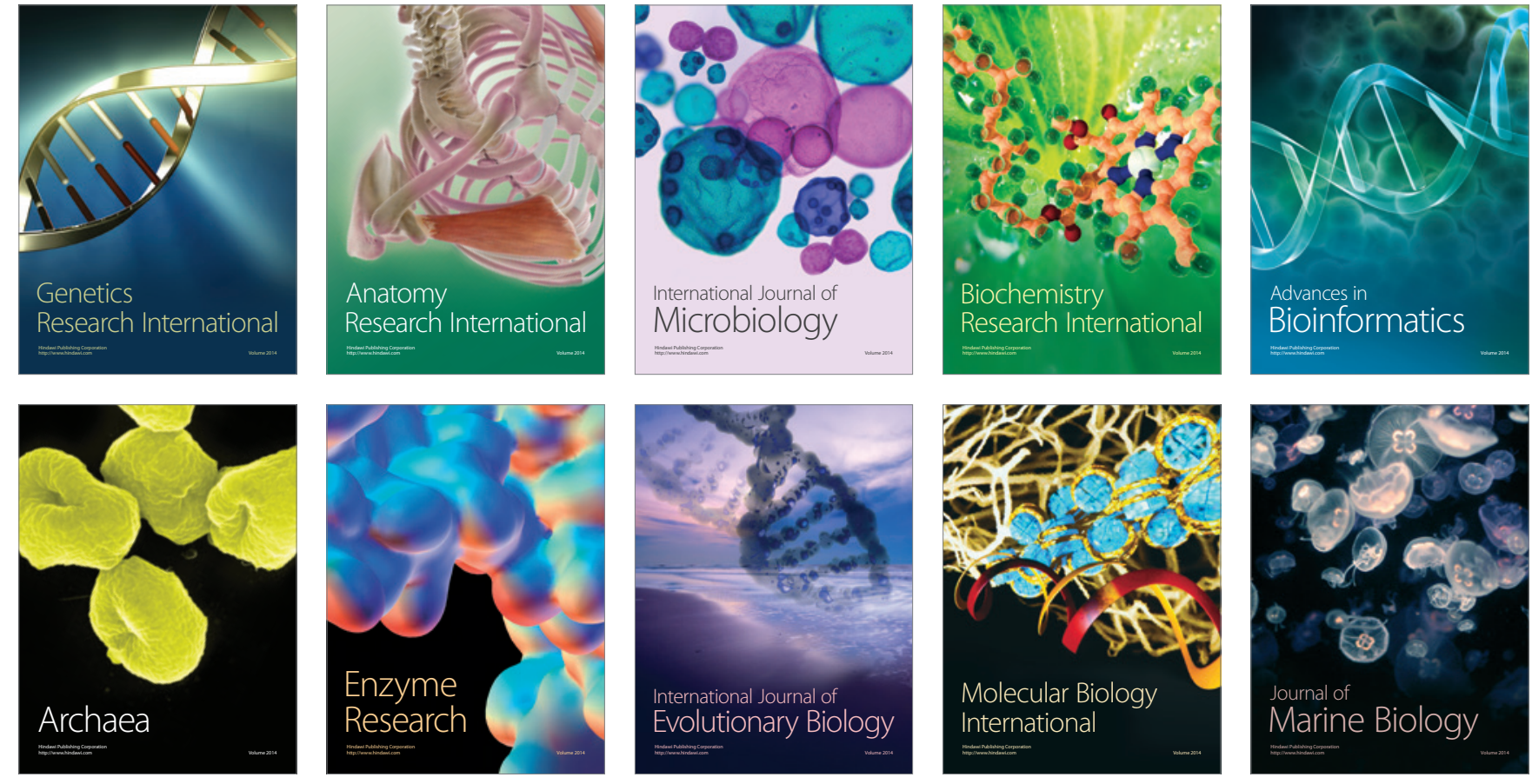